\title{
Exploring the Critical Success Factors of Virtual-Agile IT Projects: A Grounded Theory Study
}

\author{
Hassan Amar and Markus Haag
}

\begin{abstract}
Business dynamics and competition have necessitated IT organizations to use virtual style of working depending on the principles of agile methods. Virtual-agile IT projects are most relevant in the context of software and web development, new product development and information system projects. Organizations where fulfilling the demands of clients, face several complexities during projects; mainly obstructed by differences in location, time, culture and working dynamics etc. This paper aims to explore some useful practices undertaken in IT industry to deal with such criticalities. The research uses qualitative methods and grounded theory methodology to generate emerging themes recognized as codes or concepts; which were further transformed into categories based on their properties and dimensions. Categories or sub-categories identified were arranged using coding paradigm which helped to identify a core-category. Consequently, the relationships were refined, arranged in a unique way in selective coding phase and a storyline was generated elaborating the relationships between various categories, sub-categories and concepts. Finally, a conclusion is made and recommendations are proposed for future research.
\end{abstract}

Index Terms-Virtual projects, agile methods, grounded theory methodology, Critical Success Factors (CSFs).

\section{INTRODUCTION}

Innovations in Information and Communication Technology (ICT) have led to flexibility, variance, swiftness and development of an enhanced communicational structure in IT organizations [1]. Software or web development and new product development projects are particularly relevant in the context of using virtual working style; thus, finding them more productive and responsive to disparities and variations [2], [3]. To facilitate virtual projects, principles of agile methodology were introduced to help completion of projects in smaller portions rather following the traditional methodologies of waterfall, spiral model etc [4]. Agile which is an iterative methodology, was originally developed for collocated projects; maintains extensive collaboration, handle variations, and provides a platform to meet the demands and satisfaction of customers comprehensively [5], [6]. There are several methods which come under the umbrella of agile methodology depending on the project specifications, settings and requirements [4], [7]. These methods include extreme programming (XP), scrum, lean development, feature driven development (FDD), Dynamic

Manuscript received August 14, 2017; revised October 5, 2017.

The authors are with University of Bedfordshire, United Kingdom (e-mail: hassan.amar1@beds.ac.uk/hazzankhan@msn.com, markus.haag@beds.ac.uk/markus.haag@web.de). systems development methods (DSDM) [5], [6], [8].

Virtual-agile projects which are the part of IT projects mainly include software or web development, information systems (IS) and new product development projects [9]-[11]. In literature, there have been different terms used against the term 'virtual-agile' i.e., distributed agile development (DAD), global software development (GSD), global IT projects, global distributed software projects (GDSP) [12]-[15]. Hence, to reduce misinterpretations and confusions, the authors of this paper have introduced a generalized term of 'Virtual-Agile' which comprises of all type of IT projects based on the virtual working format and agile methodology. Virtual-agile IT projects comprises of several diversities in terms of geographical location, culture and ethnicities [16], [17] therefore, the team members networked together have different intentions and interests, desire for career growth and requirements for training on new technologies [7], [18], [19]. When the projects evolve over time, uncertainties grow as the team members interact and indulge, thus giving rise to dynamic challenges and complexities [4].

There have been several studies highlighting challenges and project managing techniques in terms of software and agile development, and virtual projects as well [16], [20]-[22] . Most of the studies share common characteristics, but are difficult to comprehend and adapt in industries because of the differences in organizational dynamics, working practices and aptitude of individuals. Therefore, this study aims to feature some useful practices based multiple realities and experiences rather being focused only on theoretical and pre-conceived terms. The researchers have been able to determine those critical practices which help to complete projects under the scope of time, cost, and quality; thus, meeting customer demands and business dynamics. Correspondingly, the main research question for this study is addressed as: What are the critical success factors of working in virtual-agile IT projects?

\section{Methodology}

This paper has used Grounded Theory Methodology [23], [24] together with Qualitative methods to explore the critical factors of success in virtual-agile IT project settings. As virtual-agile project environments are dynamic, competitive and rely on the use of innovative technological sources, several new complications and issues may rise which need to be studied from a different perspective [2], [25].

The reason for choosing the grounded theory methodology is based on the justification: 1) there have been several studies previously in the area which mainly focuses on confirmation and verification of pre-existing ideas 2) 
Literature to date mainly depends on primitive theories. Therefore, there is a need for novel theories and frameworks which could consider a distinct viewpoint on the subject area of virtual-agile projects 3) grounded theory methodology based on the principles of theoretical sampling and constant comparison also allows space for the researcher to be flexible and open towards the findings emerging from data [24], [26], [27].

Following an interpretivist paradigm, the researchers socially constructed their findings by reflecting multiple realities of successful exercises that are being practiced in IT industry or organizations [24], [26]. Focused primarily on a semi-structure interview format, the researchers conducted 18 interviews from professionals and practitioners in several different countries, namely United Kingdom, Canada, Germany, UAE, Kuwait and Pakistan.

\section{DATA COLLECTION AND ANALYSIS}

In grounded theory research, the data collection and analysis overlaps at several stages as the researcher collects data, analyze it and then goes back into the field for collecting more data. Procedures of theoretical sampling and constant comparison thus facilitated the data collection and analysis phases, and then deductions were made with the support of literature. Memo-writing process facilitated to draft the record the ideas and opinions which came during analysis.

\section{A. Theoretical Sampling and Constant Comparison}

Grounded Theory Methodology specifies a criterion for selecting participants by theoretically choosing them. Strauss and Corbin [23], [24] explains that theoretical sampling is a procedure in grounded theory study that is used to exploit the opportunities thus identifying the deviations among concepts, and then densifying the categories in relation to its properties and dimensions.

Glaser and Strauss [28], the discoverers of grounded theory methodology recognized theoretical sampling as the process of collecting data for developing theory, whereby the researcher together gathers, codes and analyses data, and decide what data to collect next and where to go to find it. Theoretical sampling does not contain full descriptive coverage, but indeed is an insight of the researcher screening through his theoretical lens [28]. The sample cannot be predetermined at the beginning, but the process allows the researcher to start data collection from some point [28] According to Strauss and Corbin [23], [24], who have a more systematic approach for conducting grounded theory study guide the researchers to have some initial insights into literature which help them to identify their focus and area of research. The initial review of literature for this study was based to identify knowledge gaps, and then further review of the literature during axial and selective coding phase guided the researchers to draft their findings.

The authors of this paper used the guidelines provided mainly by Glaser and Strauss [28] and Strauss and Corbin [23], [24] for data collection and analysis. Constant comparison of data was one of the principles which helped the researchers to identify where to go and what to collect next. Mainly dependent on the semi-structure interviews, the researchers conducted 18 interviews from different professional and practitioners who were or currently a part of virtual-agile IT projects. The interviews were conducted to get multiple insights and explore critical practices which help to complete projects on time. Cavana et al. [29] highlighted that Interviews in qualitative methods are unique form of revealing rich, rigorous and complex knowledge from a participant. Further, supporting the argument of Cavana et al. [29], Willis [30] signifies the role of interviews and suggests that

'Your view of the world plays an important stance for preparation of the interviews within the area of research being conducted. Choosing the right interviewee, designing of questions, structuring the interview and then interpretation of the data are some of the essential elements'.

Strauss and Corbin [23], [24] and Creswell [31] have also acknowledged the interviews strategy in grounded theory study by suggesting that interviews may vary in different boundaries, different experiences; from person to person and situation to situation. Though, grounded theory is compatible with a wide-ranging data collection practices, the researchers decided to use semi-structured type of interviewing technique for data collection as it is considered a useful practice to get in-depth knowledge and information. Secondly, to record wide-ranging perceptions, the researchers considered to undergo interviews in different regions as virtual-agile projects comprises of various people around the globe. It might help the researchers to recognize multiple factors of dealing with such projects. Some of the interviews were conducted face-to-face, some of them were through video conferencing and few were telephonic. Creswell [32] and Lincoln and Denzin [26] have advised that semi-structured style interviews are relevant to the settings of grounded theory; supports in the development of the emerging theory, as codes and categories evolves during and with respect to the number of interviews being conducted. As previously mentioned, a total of 18 interviews were conducted for this study. The researchers used multiple versions of interview questions to ensure rigor in methodological process. The process of constant comparison and theoretical sampling facilitated to determine the future participants and what to inquire in the forthcoming interviews. The initial interviews questions were like

'What are the challenges and issues faced during such projects?'

\section{'How those challenges and issues were managed?'}

Similarly, after initial analysis the questions were revised and based around the emerging categories and concepts like, 'What is the importance of communication in virtual-agile projects?'

'How do hiring skilled individuals reduces chances of failure?'

'Why knowledge transfer is important between the teams?'

\section{B. Memo-Writing}

Memo-writing is the process of writing down ideas and opinions about codes and categories generated; the process defines the relationship, specify properties and helps in developing a formal theory [23], [24], [28]. The process of memo-writing has been extensively used by the researchers during data collection and analysis stages. The researchers 
gave priority to writing memos during coding phase as it is the process of noting down emerging ideas against codes or categories identified. Data is always there to be analyzed afterwards but ideas are fragile, when they hit the mind, they should be noted down. The memos can be modified as more information or new concepts are emerging from the data. The memos at a later stage of the research were used in drafting the findings/theory and writing up the discussion sections.

\section{Open Coding}

Open Coding is the initial analysis phase in which the codes or concepts were identified from the data and based on their properties and dimensions further grouped into categories [23], [24]. Strauss and Corbin [24] identify properties as the features of a category; the description of which outlines and offers association and relationship. In addition, Creswell [32] have defined properties as the features that are shared to all the ideas or concepts in the category. Open coding for this research study began with line-by-line, word-to-word or phrase-by-phrase reading of the data (transcripts) which helped to identify emerging codes and concepts [26], [31].

TABLE I: OPEN CODING (CODES AND CATEGORIES)

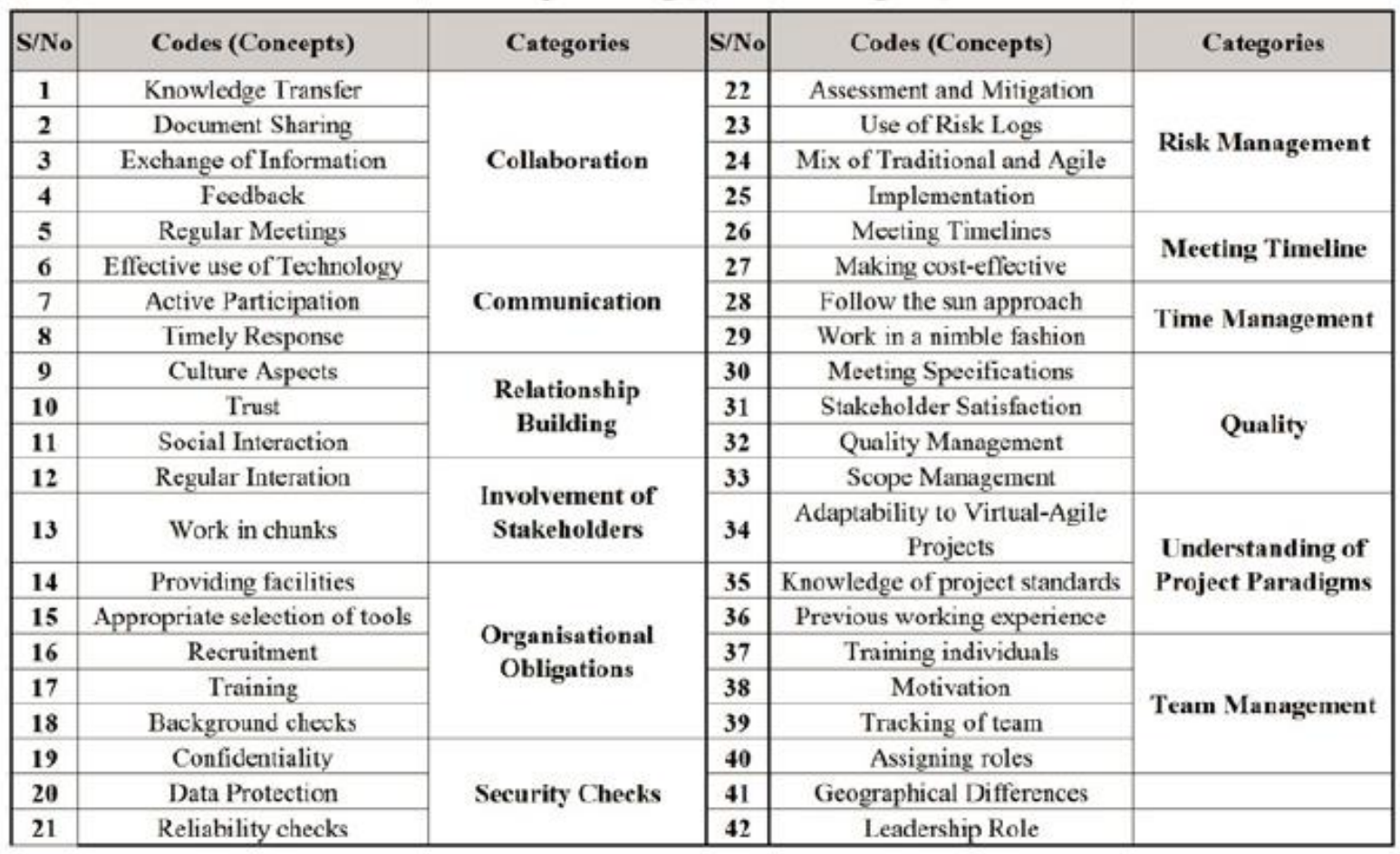

After analyzing first 3 interviews, the researchers applied the principles of theoretical sampling and inquired the questions of the data (as mentioned in sub-section A) to facilitate constant comparison based on the recommendation of Strauss and Corbin [24]. Constant comparison between newly and previously collected data assisted the grouping of numerous codes into categories. The questions which the researchers inquired to themselves during analysis of data were like 'What makes this event or action similar to the preceding one?' 'What was the response of this participant in comparison to previous participant for this particular event or action? These types of questions facilitated the process of constant comparison.

A total number of 152 open codes and 18 categories were generated during analysis. Table I shows only 42 codes and 12 categories. It is important to note here that only those codes and categories are considered in which the saturation was observed i.e., confirmed by multiple participants. Additionally, several codes were having the same meaning or properties were merged and rests of them were eliminated (usually done in selective coding).

Categories which were initially developed were refined in the following interviews depending upon the emerging nature of the data. Refinement of categories was based on reflecting several meanings of the data. Memo-writing was concurrently taking place on the themes emerging from the data, replicated the ideas in relation to the categories being developed and refined [23], [24].

\section{Axial Coding}

The next stage of coding was the axial coding. Axial coding is the phase in which the categories from the open coding phase are refined with the help of coding paradigm creating a unique relationship among them. It is important to note that the process of open and axial coding overlaps at several stages; as the researcher collects data, analyses, generates codes and categories, and then plot against the coding paradigm [31], [32]. The categories generated were organised in coding paradigm using the literature and going through the data (transcripts) again and again. According to Strauss and Corbin [24], coding paradigm consists of unique characteristics. These characteristics were used as the basis for generating a core-category in selective coding (next stage). A detailed coding paradigm has been shown in Fig. 1. This coding paradigm has been developed on the basis of Table I; which includes codes and categories. The central phenomenon identified was 'Successful ways to deal with issues and challenges'. 
- Causal Conditions - These are the conditions that impact the central phenomenon, events, incidence and happenings.

- Phenomenon - The main idea or thought, occasion, occurring incidence about which a group of activities or teamwork are focused at managing or to which the group of movements is linked.

- Context - Explains the settings in which the events occurred
- Intervening Condition - These are conditions that figure out, assist or force the strategies that occurs with a context

- Strategies - Actions undertaken to accomplish, manage or respond to a phenomenon under group of observed conditions

- Consequences - The outcomes as a result of action or interactions or outcomes as a result of applying strategies.

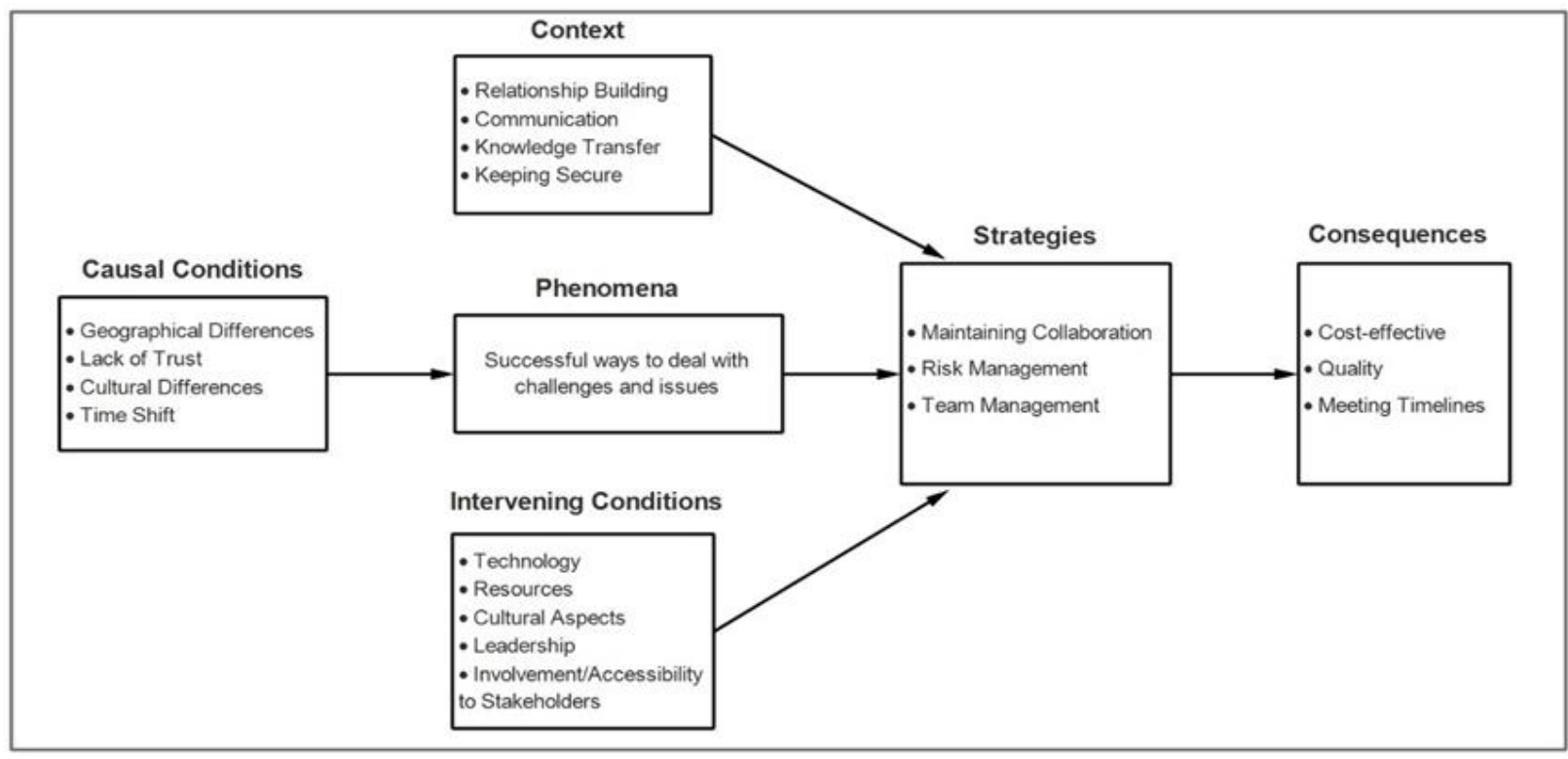

Fig. 1. Coding paradigm (axial coding).

\section{E. Selective Coding}

The purpose of selective coding is to recognise a core-category in relation to the identified phenomenon; for which the researcher produces a justification in relation to the emergent theory [31]. In selective coding, a core-category of 'collaboration' was identified by going through the literature and review of data as shown in Fig. 2. A storyline was outlined around the core-category identifying the relationships between previously refined categories, sub-categories and concepts extracted from Table I. The data was reviewed again and re-arranged in a unique way to represent them in the form of conceptual framework; connecting all the categories, sub-categories and concepts. The storyline was developed on the significance of collaboration in virtual-agile IT projects, and how different factors contribute and support to maintain collaboration. Explanation of findings was undertaken using literature, memos and consulting the data again. The core-category united all the categories to provide a justification for the phenomenon being studied. Following Strauss and Corbin's [23], [24] guidelines were applied to identify the core-category,

- It should be central; all the major or minor categories can be linked to it

- It should occur regularly in the data; meaning that all the events or actions in the data should point towards that concept

- The description should emerge itself rather than forcing the concepts
- The name used to explain the core category should represents a whole picture of it, so that it can be used for further research in other substantive areas.

\section{FINDINGS AND DISCUSSION}

According to Strauss and Corbin [23], [24] and Creswell [31], [32], the use of literature helps to verify the relationships between the developed categories, sub-categories and concepts. A storyline has been produced which helped to describe and justify the relationships between each category.

Memos which were produced concurrently during analysis provided a significance support in theory generation and write-up. Fig. 2 represents the emerging categories which are further related to their sub-categories or concepts. Big oval shapes represent main categories and small oval shapes represents sub-categories. The concepts or codes are attached to the categories and sub-categories. The core-category of collaboration represents as the major action or phenomena that is required to be undertaken in virtual-agile IT project environments; thus, providing assistance to deal with several challenges and issues, and complete projects successfully.

Maintaining an effective collaboration in virtual-agile projects is of key significance as several aspects or factors are linked to collaboration [33]. As virtual-agile projects are operated from different locations and time zones, keeping an effective collaboration reduces feeling of isolation and helps to maintain a good relationship between team members [34]. 
The category of collaboration is linked to further four sub-categories which include further concepts or codes as illustrated in Fig. 2. Those sub-categories are

- Knowledge Transfer

- Communication

- Relationship Building

\section{- Involvement of stakeholders}

Knowledge transfer process in virtual-agile project environments is of significance as it helps the team members to exchange information, share documents and give/get feedback in relation to the tasks performed. According to Reed and Knight [16], insufficient knowledge transfer leads to misinterpretation and misunderstanding and leaves a negative impact on capabilities on team members. During the data collection, one of the participants highlighted that knowledge transfer is more immediate type of thing which needs to be frequent in such projects'. Similarly, another participant mentioned that 'Sharing of knowledge ramp up the projects and help to understand tasks from multiple perspectives'. Knowledge transfer in virtual-agile projects is different to traditional projects as the only medium for exchanging information and sharing documentation is dependent upon effective use of technology [7], [35].

Similarly, effective communication plays an imperative part in maintaining collaboration. It is important to note that in Fig. 2, effective use of technology is an emerging concept of the sub-category communication; but as mentioned above effective use of technology is also required for sharing knowledge or information within the virtual-agile project teams. According to Strauss and Corbin [24], all the categories, sub-categories and concepts have a unique relationship in between that contributes to the core-category.

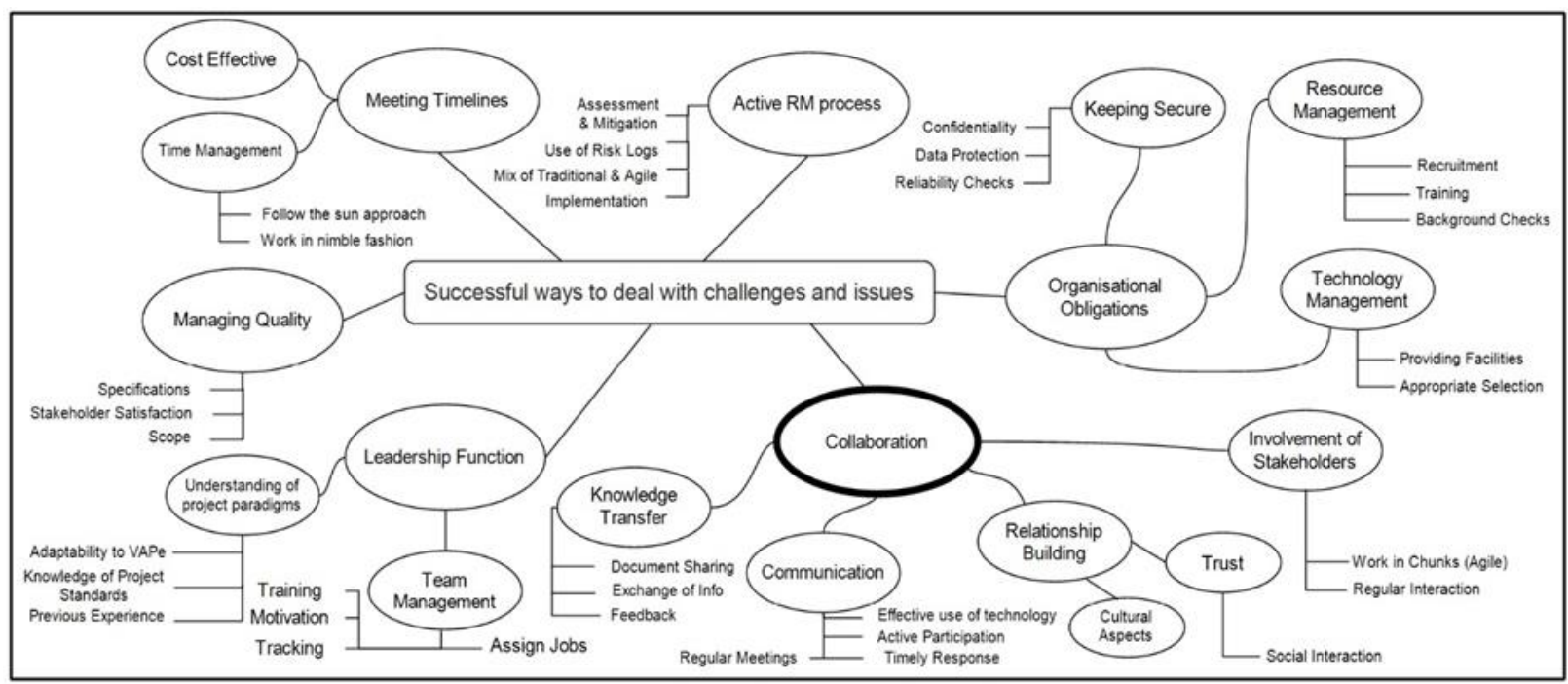

Fig. 2. Conceptual model (selective coding).

Additionally, active communication requires participation, timely response and regular meetings between the project teams. One of the interviewee mentioned that 'For keeping a persuasive communication level, team members and project manager should be active, attend meetings, know which medium to use for communication and respond timely to the queries. This can be done with having a support system using technology effectively which may assist team members completing their assignments timely'.

Building of relationship also contributes to maintain acceptable level of collaboration. Relationship building is difficult in virtual-agile projects as most of the team members are unknown to each other and therefore could not develop trust which is a key factor (minor category) of relationship building. As the team members are distributed or dispersed,

Social interaction between the teams is limited. Cultural differences may also act as hurdle in building of relationship. Cultural differences can be the differences in language, working practices or behavior. According to Cramton and Webber [21], strong relationship between geographical distributed teams helps to resolve conflicts and exchange knowledge which refines the capabilities of team members. A team having good relationship is more confident and positive to all the challenges of virtual-agile project environments. In this respect, a participant mentioned 'What lags behind is the soft element within the project or program that builds the relationship and trust within the project team'. Being anonymous to each other, soft skills helps to build a relationship, reduce conflicts and supports in nourishing collaboration [15].

The authors also identified that involvement of stakeholders for having a strong collaboration is necessary in virtual-agile format as the project is performed into little pieces and then requires regular interaction of stakeholders so that the next stage or level of the project can be proceeded. One of the participants stated that 'It is the beauty of agile that gives an opportunity to stakeholders to interact frequently and decide upon the work for next stages'. Stakeholders includes project team, board of directors, sponsors, customers/clients or individuals or organizations directly or indirectly linked to the project.

Likewise, one participant while signifying the role of stakeholders mentioned that 'Accessibility to stakeholders at several stages of the projects helps to understand the scope and maintain high collaboration level in project'.

\section{A. Organizational Obligations}

To deal with project environment ingeniously, organizations undertaking such kind of projects should fulfill their obligations in terms of managing resources, providing appropriate technology and drafting precise guidelines to 
ensure maximum security and confidentiality of data.

\section{1)Technology \& resource management}

Technology has a substantial part in overall collaboration. As the virtual-agile teams are connected over information and communication technologies (ICT), selection and provision of appropriate technologies including high speed connectivity, latest personal computers, licensed software, high definition audio-visual conferencing tools etc are obligations of an organization.

Training of project teams or individuals is another aspect which has been highlighted by many participants who were the part of this research study. Virtual-agile projects due to its complex nature, requires training on specific tools and technologies, and code of conduct which is the obligation of resource management department. According to Malhotra et al. [18], providing training to individuals or teams is wide-ranging responsibility of organizations to ensure the teams or personnel being a part of such projects fully understand their roles, mission and goals. In terms of software or web development, some participants mentioned that Scrum Master plays a key role in the training and development of individuals where he trains the teams on different jobs, tasks and discuss regulations. Hiring skilled individuals is another aspect where project managers or organizational face difficulties. In terms of resource management, organizations should select and recruit individuals or teams based on criteria and merits to ensure maximum output in a project. During an interview, one of the participants mentioned that 'Finding right individuals for such projects becomes very difficult when organizations are operating from different regions; on one side, they focus on being productive and the other side is to secure data, so they do run background checks and look into their CVs'. It is imperative in virtual-agile projects that the people who are integrated together should be able to understand the nature of the projects and their roles. Team members should know that by maintaining high collaboration, the project can bring productive results. One of the participants in this regard mentioned that 'Virtual-agile projects are like establishing a new relationship between unknowns or chosen people. Now it's up to the individuals how they manage their communication level; definitely for this they need to skilled and properly trained'

\section{2)Keeping secure}

One of another most crucial factor for managing the virtual-agile project successfully is how the organizations keep their projects secure. Organizations need to elaborate guidelines or terms and conditions for the team members who are the part of the project. A participant mentioned that 'One of the biggest problems in virtual-agile projects is the confidentiality of data linked with Public sector projects. That is the reason governments take reliability checks before giving the projects to organizations'. Similarly, the people who are part of such projects need to be credible before the organizations hire them. They may consider their backgrounds checks, previous experiences and profile before formally elaborating the project scope and objectives.

It is imperative to note here that the data interpretation represents overall emerging themes (successful working practices) of diverse types of agile methods which includes scrum, lean development, feature driven development (FDD), extreme programming (XP), dynamic system development method (DSDM) [5], [6].

Critical success factors have been extracted as common practices among all the agile methods which are essential to complete projects successfully. There can be several technicalities in agile methods, but the researchers have focused on positive practices from the managerial and administrative point of view. These successful practices (emerging themes) are applicable to all projects following aforesaid agile methods.

\section{B. Active Risk Management Process}

Another key factor which has been identified is an active risk management process. By active, the researchers mean to say that the process which is in process and functional. This overall theme has been identified in the data from view of different participants who believe that risk management process is one of the key fundamentals leading a project to success or failure. Most of practices shows that risk management process is not lively as it should be. They ignore active functionality of this process and then have to face consequences at some point. Though risk management is a part of every project, but in terms of virtual-agile projects, it is of more significance due to various factors like time shift, difference in geographic, accessibility to stakeholders and maintaining quality parameters. According to one of the participants 'Risk management in projects is now just a checkbox exercise which shouldn't be of such low standing in virtual-agile projects as it directs organizations and project teams, and produce awareness for future projects'.

Risk management for virtual-agile projects is performed in several ways. Usually the identification and assessment of risks in performed in daily, sprint or scrum meetings [37], [38]. A diary is maintained and risks are updated throughout the project life cycle. It is important to note that several participants mentioned that risk management process for virtual-agile projects is performed using a mix of traditional and agile approaches to ensure maximum control over uncertainties. Usually in terms of using specific tools, the project stakeholders discuss the likelihood vs impact of the event, and determine ownership and action strategies. Risk management process occurs throughout the project and logs created help organizations to decide upon certain strategies for forthcoming projects. Participants also enforced that active risk management process needs participants or extensive involvement of stakeholders. The discussion or meeting log should be acknowledged to all kind of stakeholders so everyone should understand what problems or events are going on within project boundaries. Here the core theme of collaboration can be linked to successful implementation of risk management process. As all the stakeholders are notified about enduring risks or uncertainties, the process is more functional, and diverse response or suggestions may help to resolve issues. The risks are more in bigger or multi-million projects where there is a regular change in scope is expected, accessibility to individuals or stakeholder is crucial, delays in sprints are expected; these sorts of complications hinder to meet the timelines and complete project under proposed costs. 


\section{Meeting Timelines}

Meeting timelines is another aspect which has been critically identified from the data. Meeting timelines include sub-category of time management and concept of cost-effective. The data suggests that time management in virtual-agile project environment is critical as individuals from various regions are connected together via information and communication technologies (ICT). For example, if one team is operating from Australia and another from USA, it becomes difficult for them to communicate due to shift in time. The participants suggested to 'follow the sun approach' in order to manage tasks and complete work on time.

Suppose, team working in Australia may handover the work to the team in USA and then this process goes throughout the project life cycle. Here, again the collaboration between the teams needs to be effective before they actually transfer the work to another team. Knowledge sharing or exchange of documents becomes a viable as the project is constrained by time. For making the virtual-agile projects cost-effective, meeting timelines helps to reduce the overhead costs that is needed whenever the project exceeds deadlines. The overhead costs may include hiring individuals or teams for a longer contract period or facing penalties. The vital point to note here that involvement of stakeholders is essential where teams, sponsors and board of directors discuss and decide upon mutual strategies to meet deadlines [38].

\section{Quality Management}

Quality management of the projects includes meeting specifications and scope and satisfaction of stakeholders who are obviously your clients or customers. As previously mentioned that agile works bits by bits and involvement of stakeholders help to reduce confusions and meet actual scope, therefore collaboration is between the customers/clients and project team is vital to meet overall specifications. The clients/customers when agrees to a certain output, the project is progressed and moved to the next stage [7], [39]. Achieving and maintaining quality in virtual-agile projects becomes difficult as the team is dispersed or located at several regions. As the project is performed using agile principles, it gives opportunity to stakeholders to interact at several stages of the project. The project stakeholders may discuss several aspects and issues making effective use of communication technologies like video conferencing, group chart or via conference.

\section{E. Leadership}

Efficient Leadership has been another characteristic which has emerged from the data which controls the projects. Project Leader is linked to all aspects of the projects but in terms of virtual-agile projects, his focus is to provide knowledge, give feedback, allocate tasks, ensure training of staff, track them and motivate them towards work. In virtual-agile projects, the data indicates that previous experience of managing such project environments counts a lot as the leader is able to manage diverse affairs. A project leader should have the knowledge and understanding of working in virtual and agile project paradigm, so that he guides his team positively. One of the participants mentioned that 'leaders in virtual-agile projects are different to traditional projects as they need to judge or estimate the caliber of teams or individuals and then allocate the tasks correspondingly'. In another viewpoint, a participant mentioned that 'leaders' knowledge makes easy to sort out work and reduce misunderstandings between the teams; though it is a shared job but leaders should and must have an upper hand as he is leading from the front'.

Collaboration again is an important fundamental which links back to effective function of leadership. Effective collaboration helps the leader to maintain the check and balance of activities, and by using technology effectively he can respond back to queries or give feedback to individuals timely. Managing virtual-agile teams is relatively challenging when teams or individuals are functioning from different geographical location. The factor of time shift makes it difficult for the team leader to correspond and communicate with individuals on time. In this regard, a participant mentioned that 'leaders' knowledge and experience helps to arrange meetings and select medium for communication; so that everything initiates and finish on time'. As virtual-agile team members are expected to be a part of another organization or projects working different jobs or assignments, it is obligatory for project leader to know about the involvements of individuals or teams, so that he may estimate how much work can be assigned. The participants suggested that leader should consult with all the internal stakeholders before taking a decision or acting upon a plan; as this helps to create a mutual understanding and reduce blame-game.

\section{CONCLUSION AND FUTURE RESEARCH}

The data analysis shows that maintaining effective collaboration is the most significant feature in order to successfully deal with challenges and issues in virtual-agile project environments. The results also suggested that the emerging themes or categories all link back to collaboration as the projects are distributed or dispersed. Collaboration affects directly or indirectly all the categories or sub-categories mentioned in Fig. 2. This study has provided in-depth analysis of factors which helps to complete the project on time. The data was collected from various professionals and practitioners which gave highlight how effectively the virtual-agile project environment be managed. The critical success factors which are identified and discussed are applicable to all type of virtual-agile projects. These are the basic practices which an organization or project leader should adhere. Future research should be conducted focusing individual category in Fig. 2 and discuss how the relationship between various categories or sub-categories changes with respect to project requirements, specifications or scope. This will provide in-depth review of categories and their relationship and variation in such project environments.

\section{REFERENCES}

[1] G. Levin and P. F. Rad, Achieving Project Management Success using Virtual Teams, Boca Raton, USA: Ross Publishing, 2003.

[2] V. Badrinarayanan and D. Arnett, "Effective virtual new product development teams: An integrated framework," J. Bus. Ind. Mark., vol. 23, no. 4, pp. 242-248, 2008.

[3] G. R. Berry, "Enhancing effectiveness on virtual teams: Understanding why traditional team skills are insufficient," J. Bus. Commun., vol. 48 no. 2, pp. 186-206, 2011. 
[4] J. Binder, L. I. Aillaud, and L. Schilli, "The project management cocktail model: An approach for balancing agile and ISO 21500," Procedia - Soc. Behav. Sci., vol. 119, no. 119, pp. 182-191, 2014.

[5] M. Pikkarainen, O. Salo, R. Kuusela, and P. Abrahamsson, "Strengths and barriers behind the successful agile deployment - Insights from the three software intensive companies in Finland," Empirical Software Engineering, vol. 17, no. 6, pp. 675-702, 2012.

[6] G. Chin, Agile Project Management: How to Succeed in the Face of Changing Projects, NewYork, USA: AMACOM, 2004.

[7] M. L. Drury-Grogan, "Performance on agile teams: Relating iteration objectives and critical decisions to project management success factors," Inf. Softw. Technol., vol. 56, no. 5, pp. 506-515, 2014.

[8] S. Lee and H. S. Yong, "Distributed agile: Project management in a global environment," Empirical Software Engineering, vol. 15, no. 2, pp. 204-217, 2010.

[9] K. M. Chudoba, E. Wynn, M. Lu, and M. B. Watson-Manheim, "How virtual are we? Measuring virtuality and understanding its impact in a global organization," Inf. Syst. J., vol. 15, no. 4, pp. 279-306, 2005.

[10] A. A. Keshlaf and S. Riddle, "Risk management for web and distributed software development projects," in Proc. 2010 Fifth Int. Conf on Internet Monit. Prot. (ICIMP), January 2010, pp. 22-28.

[11] P. K. Vijayaraghavan, S. Sundararajan, and M. Bhasi, "Case study on risk management practice in large offshore-outsourced Agile software projects," IET Softw., vol. 8, no. 6, pp. 245-257, 2014.

[12] O. K. D. Lee and D. V. Baby, "Managing dynamic risks in global it projects: Agile risk-management using the principles of service-oriented architecture," International Journal of Information Technology \& Decision Making, vol. 12, no. 6, pp. 1121-1150, 2013.

[13] E. Hossain, P. Bannerman, and D. Jeffery, "Scrum practices in global software development: A research framework," Prod. Softw. Process, pp. 88-102, 2011.

[14] J. S. Persson, L. Mathiassen, J. Boeg, T. S. Madsen, and F. Steinson, "Managing risks in distributed software projects: An integrative framework," vol. 56, no. 3, pp. 508-532, 2009.

[15] S. V. Shrivastava and U. Rathod, "Categorization of risk factors for distributed agile projects," Inf. Softw. Technol., vol. 58, pp. 373-387, 2015.

[16] A. H. Reed and L. V. Knight, "Effect of a virtual project team environment on communication-related project risk," Int. J. Proj. Manag., vol. 28, no. 5, pp. 422-427, 2010.

[17] P. Pinjani and P. Palvia, "Trust and knowledge sharing in diverse global virtual teams," Inf. Manag., vol. 50, no. 4, pp. 144-153, 2013.

[18] A. Malhotra, A. Majchrzak, and B. Rosen, "Leading virtual teams," Acad. Manag. Perspect., vol. 21, no. 1, pp. 60-70, 2007.

[19] J. O. Oguntebi, "Creating effective global virtual teams: A transactive memory perspective," Diss. Abstr. Int. Sect. B Sci. Eng., vol. 71, p. 281, 2009.

[20] W. F. Cascio, "Managing a virtual workplace," Acad. Manag. Perspect., vol. 14, no. 3, pp. 81-90, 2000.

[21] C. D. Cramton and S. S. Webber, "Relationships among geographic dispersion, team processes, and effectiveness in software development work teams," J. Bus. Res., vol. 58, no. 6, pp. 758-765, 2005.

[22] R. Paul, J. R. Drake, and H. Liang, "Global virtual team performance The effect of coordination effectiveness, trust, and team cohesion," IEEE Trans. Prof. Commun., vol. 59, no. 3, pp. 186-202, 2016.

[23] A. Strauss and J. Corbin, Basics of Qualitative Research: Grounded Theory Procedures and Techniques. Thousand Oaks, USA: Sage Publications, 1990

[24] A. Strauss and J. Corbin, Basics of Qualitative Research. Grounded Theory Procedures and Techniques, 2nd ed. Thousand Oaks, USA: Sage Publications, 1998.

[25] S. Matalonga, M. Solari, and G. Matturro, "Factors affecting distributed agile projects: A systematic review," Int. J. Softw. Eng. Knowl. Eng., vol. 23, no. 9, pp. 1289-1301, 2013.

[26] S. Y. Lincoln and N. K Denzin, Introduction: The Discipline and Practice of Qualitative Research, 3rd ed. Thousand Oaks, USA: Sage, 2005.
[27] S. M. Kolb, "Grounded theory and the constant comparative method Valid research strategies for educators," J. Emerg. Trends Educ. Res Policy Stud., vol. 3, no. 1, pp. 83-86, 2012.

[28] A. Strauss and B. Glaser, The Discovery of Grounded Theory: Strategies for Qualitative Research, New York, USA: Aldine, 1967.

[29] Y. R. Cavana, L. B. Delahaye, and U. Sekaran, Applied Business Research: Qualitative and Quatitative Methods, 1st ed. USA \& Australia: John Wiley \& Sons, 2001.

[30] J. Willis, Foundations of Qualitative Research: Interpretive and Critical Appraoches, Thousand Oaks, USA: Sage, 2007.

[31] J. Creswell, Research Design: Qualitative, Quantitative, and Mixed Approaches, Thousand Oaks, USA: Sage, 2009.

[32] J. Creswell, Qualitative Design and Research Design, 2nd ed. London, UK: Sage, 2007.

[33] G. Papadopoulos, "Moving from traditional to agile software development methodologies also on large, distributed projects," Procedia - Soc. Behav. Sci., vol. 175, pp. 455-463, 2015.

[34] B. L. Kirkman, B. Rosen, C. B. Gibson, P. E. Tesluk, and S. O. McPherson, "Five challenges to virtual team success: Lessons from Sabre, Inc.," Acad. Manag. Exec., vol. 16, no. 3, pp. 67-79, 2002.

[35] I. Bardhan, V. V. Krishnan, and S. Lin, "Team dispersion, information technology, and project performance," Prod. Oper. Manag., vol. 22, no. 6, pp. 1478-1493, 2013.

[36] A. Lamersdorf, J. Munch, A. Fernandez-del Viso Torre, and C. R. Rebate Sanchez, "A risk-driven model for work allocation in global software development projects," in Proc. 2011 6th IEEE Int. Conf. Glob. Softw. Eng. (ICGSE), 2016, pp. 15-24.

[37] E. E. Odzaly, D. Greer, and D. Stewart, "Lightweight risk management in agile projects," in Proc. Int. Conf. Softw. Eng. Knowl. Eng. SEKE, vol. 2014, 2014, pp. 576-581.

[38] A. Stare, "Agile project management in product development projects," Procedia - Soc. Behav. Sci., vol. 119, pp. 295-304, 2014.

[39] L. Sarigiannidis and P. D. Chatzoglou, "Quality vs risk: An investigation of their relationship in software development projects," Int. J. Proj. Manag., vol. 32, no. 6, pp. 1073-1082, 2014.

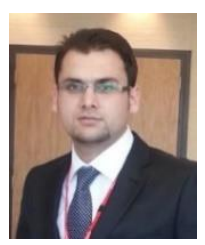

Hassan Amar is an electrical engineer by profession. The author has vast experience of working in an electronic manufacturing industry where he functioned mainly in a project manager role. Moving from engineering to project management, the author firstly completed his master's degree and now he is in final stages of his doctorate research in the field of risk management in IT Projects.

Hassan Amar obtained the bachelor of electrical engineering (telecommunication) in Air University, Islamabad, Pakistan. He obtained the masters of science in project management (Commendation) from University of Bedfordshire, Luton, United Kingdom. He obtained the doctor of philosophy in IT project management from University of Bedfordshire, Luton, United Kingdom.

He together with industrial experience has also attained rich experience of Academia during his doctorate degree. He has been working for Strategy and Management Department of University of Bedfordshire since October 2015 where he has functioned mainly as visiting lecturer and consultant. Currently, he is serving the department as unit leader for a project management unit, where he is solely responsible for the unit from design to delivery.

Additionally, the author's main interest is in qualitative research where he is currently developing a theory for managing risks and issues in virtual-agile IT projects. He has recently presented a full paper in British Academy of Management, Warwick, Sep 2017. Consequently, the author's paper was also acknowledged at Management International Conference, Italy, May 2017. The author's industrial background and mindset has aided him to bring the practical world into literature and thus, he further aims to contribute in the field of Academia. 